\title{
Polarized Neutron Reflectivity Investigation of Periodic Magnetic Rings
}

\author{
F. Y. Ogrin ${ }^{1}$, S. M. Weekes ${ }^{1}$, B. Cubitt ${ }^{2}$, A. Wildes ${ }^{2}$, A. Drew ${ }^{3}$, C. A. Ross ${ }^{4}$, W. Jung ${ }^{4}$, R. Menon ${ }^{5}$, and \\ B. Toperverg $2,6,7,8$ \\ ${ }^{1}$ School of Physics, University of Exeter, Exeter EX4 4QL, U.K. \\ ${ }^{2}$ Institut Laue-Langevin, Grenoble BP 156-38042, France \\ ${ }^{3}$ Group FK, Department of Physics, University of Fribourg, CH-1700 Fribourg, Switzerland \\ ${ }^{4}$ Department of Materials Science and Engineering, Massachusetts Institute of Technology, Cambridge, MA 02139 USA \\ ${ }^{5}$ Research Laboratory of Electronics, Massachusetts Institute of Technology, Cambridge, MA 02139 USA \\ ${ }^{6}$ Petersburg Nuclear Physics Institute, Gatchina, Russia \\ ${ }^{7}$ Institut Laue-Langevin BP 156 - 38042 Grenoble Cedex 9, France \\ ${ }^{8}$ Department of Solid State Physics IV, Ruhr-Universitaet-Bochum, Bochum D-44781, Germany
}

\begin{abstract}
Magnetic ring structures are of particular interest, due to stable remanent configurations such as the vortex state, which have potential in future magnetic random access memory (MRAM) applications. Here we report results on the first experimental study of periodic ring arrays using off-specular polarized neutron reflectivity (PNR). The experiments were performed on a $7.5 \mathrm{~mm} \times 5 \mathrm{~mm}$ NiFe array with an average ring diameter of $3.2 \mu \mathrm{m}, 0.7 \mu \mathrm{m}$ width, $25 \mathrm{~nm}$ thickness, and separation of $5.6 \mu \mathrm{m}$. Using polarization analysis, a spin dependent diffraction of reflected and transmitted neutron beams were investigated as a function of external applied field.
\end{abstract}

Index Terms-Magnetic domains, magnetization reversal, polarized neutron reflectivity.

\section{INTRODUCTION}

$\mathbf{R}$ ING STRUCTURES have attracted attention recently because of the existence of flux-closed magnetic configurations, vortex states (see Fig. 1), in which the magnetization runs circumferentially around the ring, either clockwise or counterclockwise [1]. Given the symmetry of the ring and periodic nature of the lattice, patterned rings are particularly interesting for diffraction experiments. The off-specular polarized neutron reflectivity (PNR) measurements can specifically provide information on the lateral correlations associated with the magnetic configuration (e.g., antiferromagnetic alignment). With this view, the off-specular PNR is unique as it can resolve the local magnetization even when the macroscopic average value is equal to zero. Patterned structures are particularly appealing for off-specular measurements, as these naturally provide the geometrical structure for diffraction. However, the off-specular diffraction experiments generally rely on large area periodicity, which has to be maintained throughout the whole area of the sample to produce well-distinguished Bragg peaks.

Previously, specular PNR has been successfully applied to study nanostructured magnetic materials [1]. By modelling the potential associated with the in-depth profile of the system complex 3-D magnetic structures could be identified. Examples include exchange biased, exchange spring systems and GMR superlattices (see for review [2]). Off-specular PNR studies have also been under focus in recent years [2]-[6], mainly in studies of correlation/disorder of magnetic domains in thin film and multilayers [3]. A limited number of experiments have been carried out on patterned systems [5], [6]. The theoretical formalism has been provided by a number of authors (e.g., [4]). The pat-

Digital Object Identifier 10.1109/TMAG.2007.893525

Color versions of one or more of the figures in this paper are available online at http://ieeexplore.ieee.org.
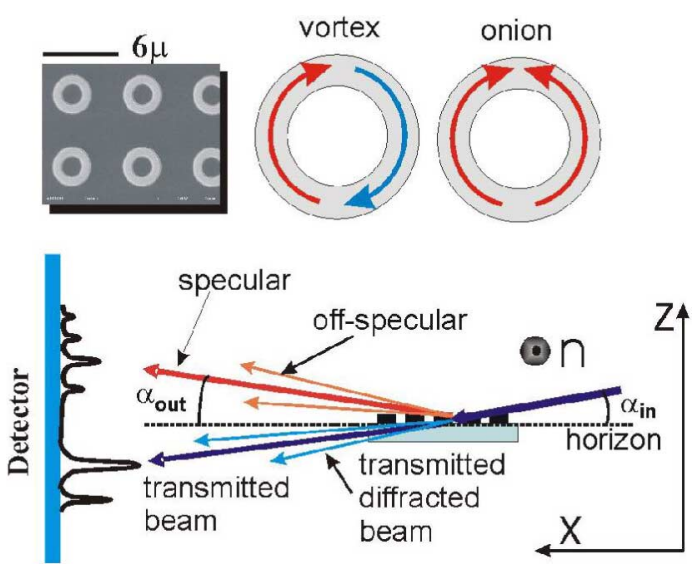

Fig. 1. Reflection and diffraction geometry of the experiment. The orientation of the applied field and polarization of the neutrons is perpendicular to the plane of the drawing. The curve on the detector plate shows a schematic variation of the measured intensity for a fixed incident angle (as shown further in Fig. 3 and 4). The insets show a SEM image of small section of the sample [7], [9], and a schematic representation of the vortex and onion states. The red and blue arrows indicate the idealistic orientation of magnetization in the rings in each case.

terned rings offer an excellent testing ground for off-specular measurements. Given the well-outlined shape anisotropy, the three distinctive magnetic configurations which can be obtained through the application of the external field are saturated, onion, and vortex. The last two (see Fig. 1) represent particularly interesting cases for scattering, because both have circumferentially aligned moments. In one case (vortex) the average moment is zero, whereas in the other (onion) there is a significant component along the field direction. From the neutron point of view, the information may be still available in the vortex state (with zero average moment) by testing the spin-flip scattering channel provided the chirality is not random.

\section{EXPERIMENT}

The samples have been fabricated specifically for PNR experiments by using zone plate array lithography [7], [9]. 
Each sample contained three patches of $2.5 \mathrm{~mm} \times 5 \mathrm{~mm}$ of patterned rings, which were closely "stitched" with a gap of approximately $50 \mu \mathrm{m}$. The ring array had dimensions of $3.2 \mu \mathrm{m}$ in outer diameter, $700 \mathrm{~nm}$ of width, and $5.6 \mu \mathrm{m}$ in center-to-center separation. The thickness of the magnetic layer was around $25 \mathrm{~nm}$. The PNR experiments were carried out at ILL facilities in Grenoble, on a high flux reflectometer D17. The experimental setup is shown in Fig. 1. The measurements were carried out in monochromatic regime, with a fixed value of the neutron wavelength $\lambda=4.5 \mathrm{~A}$. The spin polarization analysis was performed using a $\mathrm{He}^{3}$ analyzer. Following well-established methodology the measurements of four channels, two non spin-flip $I^{++}, I^{--}$, and two spin-flip $I^{+-}$and $I^{-+}$, have been undertaken. Here $I$ designates the intensity of the scattered signal, and the position of the signs ( + or - ) indicates the initial and the final polarization of the neutron. In the first case, only those neutrons are counted whose polarization has not been modified (non spin-flip channels). In the second case, conversely, only those neutrons whose polarization has been changed from "up" to "down" or "down" to "up" (spin-flip channel) are detected. The monochromatic measurements have been performed using the following approach. First, a scan of "theta-two-theta" dependence (Fig. 2) has been undertaken in order to determine the incident angles $\alpha_{\text {in }}$ for which the magnetic asymmetry $R=\left(I^{++}-I^{--}\right) /\left(I^{++}+I^{--}\right)$had the maximum variations corresponding to the off-specular Bragg diffraction. Fig. 2(b) shows an intensity map for the magnetic asymmetry R taken for a range of incident angles $\alpha_{\mathrm{in}}$. Second, an incident angle was fixed and the intensities for the four channels have been measured as a function of the output angle $\alpha_{\text {out }}$. Fig. 3 shows the result of the measurement taken at $\alpha_{\text {in }}=0.8$ degrees and at an external applied field of 100 Oe. The same measurements were then repeated for different applied fields to map out the behavior of the four intensities for different points in the hysteresis loop. Fig. 4 demonstrates the dependence of the magnetization component parallel to the applied field, as was derived from the asymmetry curves produced for these points (see discussion above). All the intensity plots have been corrected according to the efficiencies of the monochromator and the $\mathrm{He}^{3}$ analyzer used in the experiment.

\section{DISCUSSION}

To understand the magnetization behavior as a function of the external field, one can consider the relative magnitudes of $I^{++}, I^{--}, I^{+-}$, and $I^{-+}$. The intensities of the non spin-flip channels is a result of the interference between the scattering from the magnetization component parallel to the field and nuclear scattering from the ring itself, whereas the intensities of the spin-flip channels describe the behavior of the component perpendicular to the field direction (which is also parallel to the neutron polarization) [8] and is purely magnetic. In Fig. 2(b), the asymmetry formed by the spin-flip channels, $I^{++}$and $I^{--}$, demonstrates a similar magnetic structure, as that defined by the contribution of the nuclear components. Fig. 4(a) shows the asymmetry $\mathrm{R}$ taken for all the detector pixels. The integral area below the curve is proportional to the value of the parallel magnetization. For comparison, Fig. 4(a) also demonstrates the case

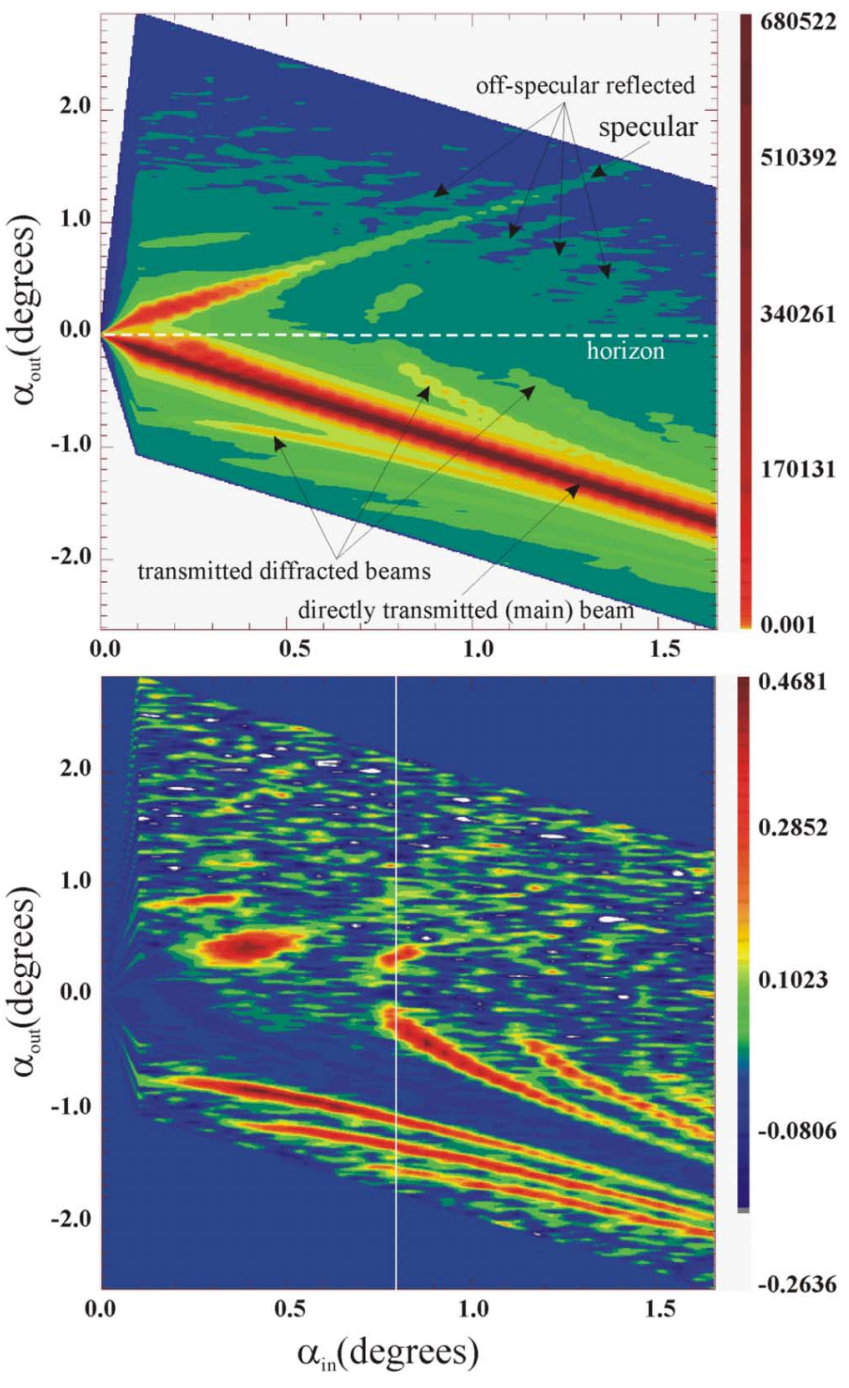

Fig. 2. Top: Reflectivity/diffraction intensity map for a theta-two-theta scan in the range of available input and output angles. The example shows a 2-D response for a $I^{++}$non spin-flip channel measured at $100 \mathrm{Oe}$. As well as the reflective specular line, measured at angle $\alpha_{\text {out }}=\alpha_{\text {in }}$, several orders of offspecular Bragg lines are clearly seen at higher and lower output angles. Bottom: The magnetic asymmetry R formed by the non spin-flip channels. In this case, the main transmitted beam is not seen as it does not contain any magnetic information, and thus is fully subtracted by the asymmetry relation. The vertical white line indicates the incident angle $\sim 0.8$ degrees, used for measurements with field dependence.

in a field of $5 \mathrm{G}$ when the parallel magnetization is zero (expected vortex state). The asymmetry is nearly zero at all angles, confirming the results produced by alternating gradient magnetometer (AGM) [Fig. 4(b)].

Interestingly, the scattering intensity of the spin-flip channels did not show any significant variation across all output angles. Both states, vortex and onion, should have a fair amount of nonzero transverse magnetization $M_{x}$, which could provide scattering potential for the spin-flip channels. In an ideal symmetric case, this would account for as much as half of the components in the perpendicular direction. However, for all the values of the external fields there was no spin-flip scattering found. If the sample in the onion state, with a major magnetization component along the direction of the applied field, the neutrons will encounter perpendicular antiferromagnetically aligned magnetic components within coherent regions of each 


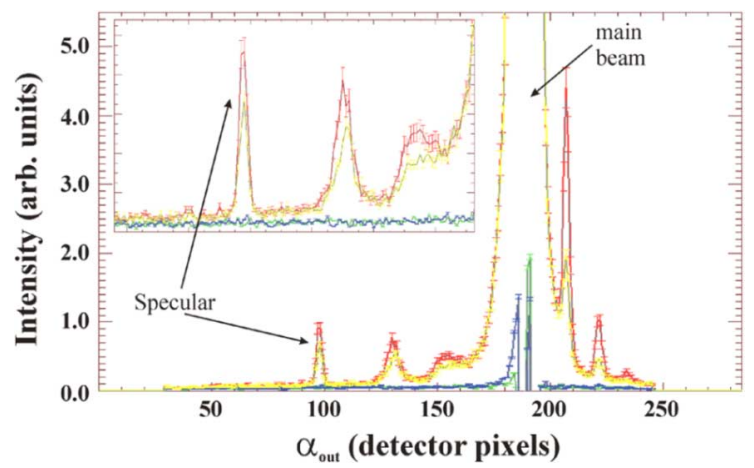

Fig. 3. A 1-D cut from the reflectivity map, taken at $\alpha_{\text {in }}=0.8$ degrees. Yellow and red: non spin-flip $I^{++}$and $I^{--}$channels; blue and green: spin-flip channels $I^{+-}$and $I^{-+}$. Applied field: $100 \mathrm{G}$. The difference between yellow and red curves demonstrates the asymmetry associated with the magnetic parallel component to the field direction. The inset shows amplified a reflectivity region with specular and off-specular diffraction peaks. The spin-flip channel signals exhibit no significant variation at neither of the reflective or transmitted diffractive peaks.
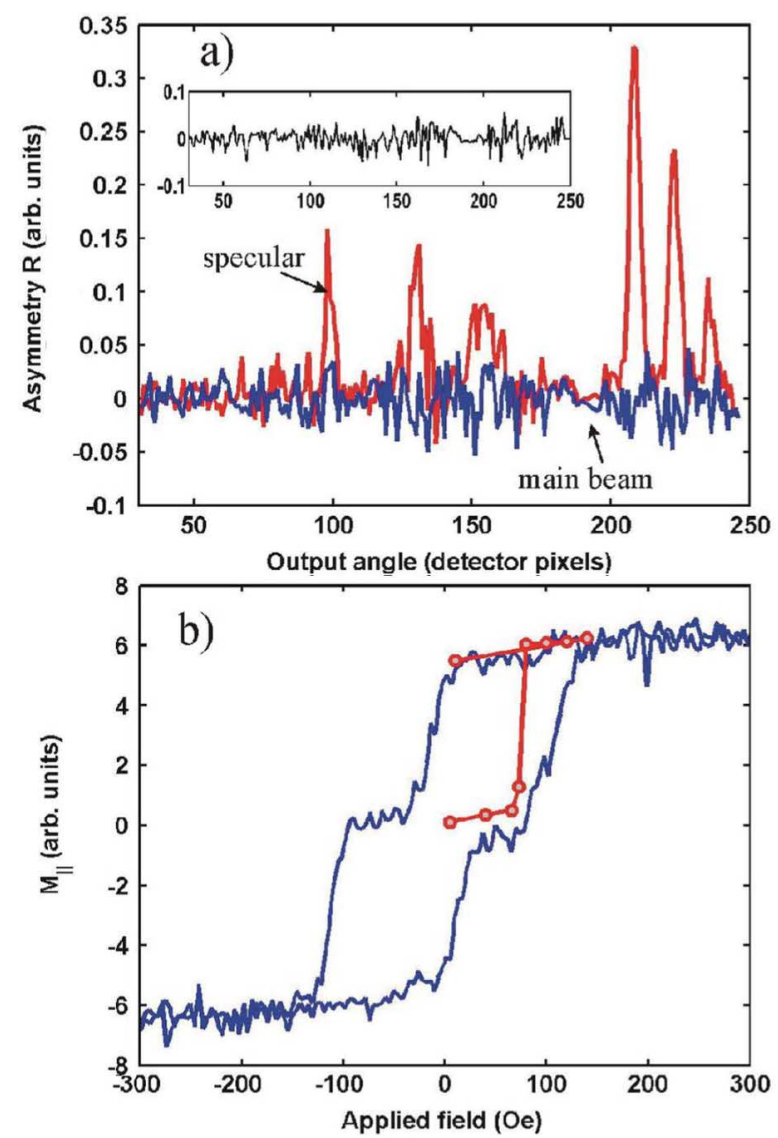

Fig. 4. (a) Magnetic asymmetry $\mathrm{R}$ taken at $\alpha_{\text {in }}=0.8$ degrees for two values of the applied field: $100 \mathrm{Oe}$ (red) and $5 \mathrm{Oe}$ (blue). Inset shows on the same scale the reflectivity intensity $I^{+-}$for the applied field of 5 Oe. (b) Magnetization component parallel to the field as measured by AGM: blue, and as derived from the asymmetry plots: red. The latter is qualitatively estimated by integrating the asymmetry curve. Both curves are normalized to each other.

ring, which would be expected to result in spin flip scattering. In the vortex state, this would be different depending on the level of correlation of the chirality or sense of the vortex rotation. If all vortices turned in the same direction at the top and bottom of each ring, the transverse moments would all point in the same direction and coherent spin flip would be expected. However, if the chirality was random, then no spin-flip signal would be seen. Although the onion state is expected to produce a nonzero scattering in the experiments, it was not possible to resolve a well-distinguished signal at any values of the applied field.

Fig. 4(b) demonstrates the comparison of the magnetization curve estimated by off-specular PNR and the results produced by AGM measurements. It can be seen that the correlation is very good, in both cases demonstrating a region with an expected vortex state formation. The PNR measurements however, demonstrate a lower switching field to the onion state. This may be a result of the different timescales of the two experiments. In PNR, the sample is at a steady field for many hours, whereas the AGM was swept at a rate of 2 Gauss per second.

\section{CONCLUSION}

We have performed off-specular PNR experiments on arrays of patterned microscopic NiFe rings. Estimating the magnitude of the magnetic asymmetry in the non spin-flip channel, a hysteretic loop has been obtained for the field dependence. The absence of any spin-flip signal at all applied field values leads us to conclude that the onion state, if it exists, has undetectable transverse magnetic components. The presence of a plateau of zero collinear magnetization components in the range $\pm 50 \mathrm{G}$ suggests strong evidence for a vortex state with random chirality.

\section{ACKNOWLEDGMENT}

The authors thank the Institute of Laue-Langevin and CCLRC facilities for providing technical and financial support.

\section{REFERENCES}

[1] F. Castano, A. Eilez, W. Jung, C. Frandsen, and C. A. Ross, "Stability of magnetic configurations in 160-520 nm diameter ferromagnetic rings," Phys. Rev. B, vol. 69, p. 144421, 2004.

[2] M. R. Fitzsimmons, S. D. Bader, J. A. Borchers, G. P. Felcher, J. K. Furdyna, A. Hoffmann, J. B. Kortright, I. K. Schuller, T. C. Schulthess, S. K. Sinha, M. F. Toney, D. Weller, and S. Wolf, "Neutron scattering studies of nanomagnetism and artificially structured materials," $J$. Magn. Magn. Mat., vol. 271, pp. 103-146, 2004.

[3] S. Langridge, J. Schmalian, C. H. Marrows, D. T. Dekadjevi, and B. J. Hickey, "Quantification of magnetic domain disorder and correlations in antiferromagnetically coupled multilayers by neutron reflectometry," Phys. Rev. Lett., vol. 85, pp. 4964-4967, 2000.

[4] B. P. Toperverg, G. P. Felcher, V. V. Metlushko, V. Leiner, R. Siebrecht, and O. Nikonov, "Grazing incidence neutron diffraction from large scale 2D structures," Physica B, vol. 283, pp. 149-152, 2000.

[5] K. Temst, M. J. Van Bael, and H. Fritzsche, "Application of off-specular PNR to measurements on an array of mesoscopic ferromagnetic disks," Appl. Phys. Lett., vol. 79, p. 991, 2001.

[6] K. Theis-Brohl, M. Wolff, A. Westphalen, H. Zabel, J. McCord, V. Hoink, J. Schmalhorst, G. Reiss, T. Weis, D. Engel, A. Ehresmann, U. Rucker, and B. Toperverg, "Exchange-bias instability in a bilayer with an ion-beam imprinted stripe pattern of ferromagnetic/antiferromagnetic interfaces," Phys. Rev. B., vol. 73, 2006, Art. No. 174408.

[7] W. Jung, F. J. Castaño, D. Morecroft, C. A. Ross, R. Menon, and H. I. Smith, "Magnetization reversal in single-layer and exchange-biased elliptical-ring arrays," J. Appl. Phys., vol. 97, pp. 1-3, 2005, 10K113.

[8] C. F. Majkrzak, "Neutron scattering studies of magnetic thin films and multilayers," Physica B, vol. 221, p. 342, 1996.

[9] D. J. D. Carter, D. Gil, R. Menon, M. K. Mondol, H. I. Smith, and E. H. Anderson, "Maskless, parallel patterning with zone-plate array lithography," J. Vac. Sci. Tech. B, vol. 17, p. 3449, 1999.

Manuscript received October 31, 2006; revised February 16, 2007 (e-mail: f.y.ogrin@exeter.ac.uk). 